\title{
«Die Betrachter zum Lachen und Nachdenken bringen»
}

\section{Matthias Scholer}

Online- und Printredaktor SÄZ

\begin{abstract}
Alternative Heilmethoden, Auswüchse des Spardrucks im Gesundheitsbereich, Digitalisierung in der Medizin - dies ist nur eine kleine Auswahl von Themen, die der Cartoonist Oswald Huber mit spitzer Feder aufs Papier bringt. Seit Anfang 2017 zeichnet der gebürtige Salzburger und emeritierte Psychologieprofessor auch für die Schweizerische Ärztezeitung.
\end{abstract}

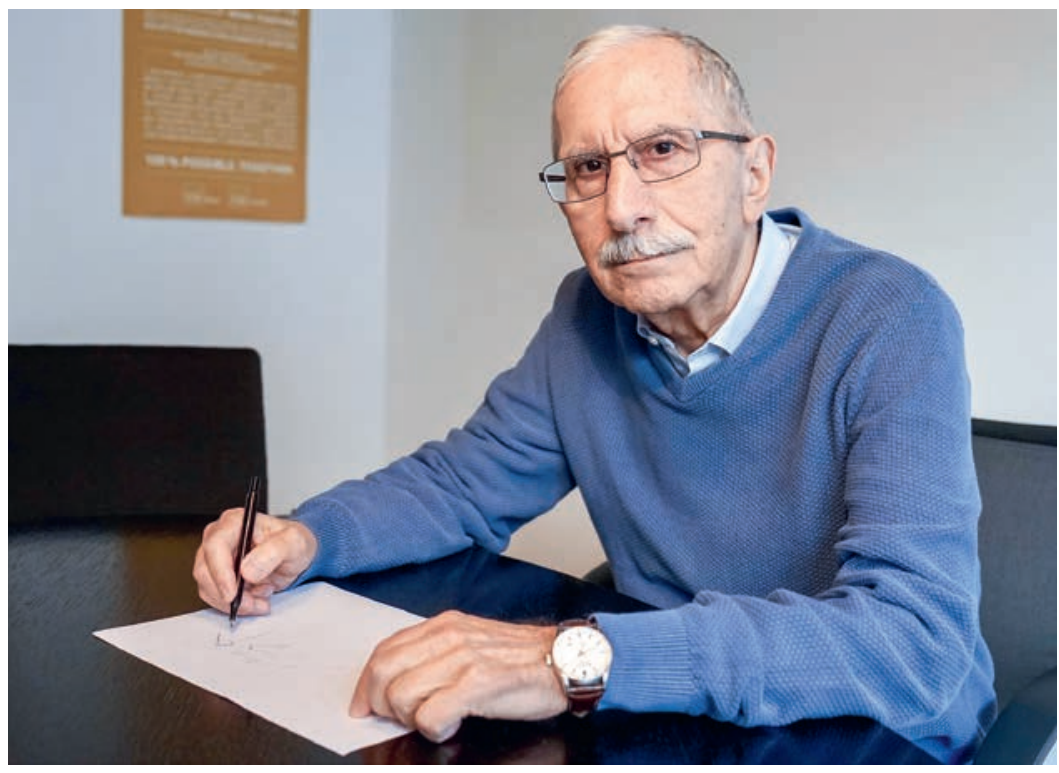

Oswald Huber zeichnet regelmässig für die Schweizerische Ärztezeitung.

Cartoons faszinieren Oswald Huber seit seiner Kindheit. Die ehemalige Besatzungsmacht USA richtete in Hubers Geburtsstadt Salzburg ein sogenanntes information center ein, in dem sich die Bevölkerung Bücher und Schallplatten ausleihen konnte. Dieses besuchte Huber bereits als Sechsjähriger regelmässig, um durch die aufgelegten Bücher zu blättern. Den kleinen Oswald beeindruckten dabei vor allem die Zeichnungen renommierter Cartoonisten wie George Price oder Peter Arno. Huber erinnert sich lachend: «Ich merkte mir die Namen nicht, weil ich nicht wusste, dass diese wichtig sind.» Erst Jahre später konnte er die Künstlernamen mit den entsprechenden Werken aus seinen Kindheitserinnerungen in Verbindung bringen.
Oswald Hubers erste Veröffentlichung seiner Zeichnungen umfasste etwa achtzig Seiten, erschien nur in einem Exemplar und war gezielt für eine Person ausgelegt: seine erste Freundin.

\section{Wegweisende Entscheidungen}

Von der Kunst zu leben, kam in der Nachkriegszeit nicht in Frage. Und auch ein Studium lag aus finanziellen Gründen nicht drin. Also musste eine solide Ausbildung gefunden werden. «Der Berufsberater empfahl mir, in der Druckbranche zu arbeiten. Diese sei ein sicherer Wert», erzählt Huber. So entschied er sich, eine Lehre als Reprofotograf zu absolvieren. «Ein absoluter Routinejob, ohne Spielraum für Kreativität», sagt der Österreicher. Doch die dadurch gewonnene finanzielle Sicherheit erlaubte es Oswald Huber später, am Abendgymnasium in Wien die Matura nachzuholen und danach an der Universität Salzburg Psychologie, Philosophie und Zoologie zu studieren. Seine akademische Karriere mündete in eine Professur im Bereich der Psychologie an der Universität Fribourg. Huber forschte hier hauptsächlich im Bereich der Entscheidungslehre. «Wir analysierten ab den 1990er Jahren Entscheidungsprozesse in Alltagssituationen", erzählt der Psychologe. In zahlreichen Forschungsarbeiten konnte Huber mit seinem Team aufzeigen, dass wir Menschen dabei nicht an exakten Wahrscheinlichkeiten interessiert sind. Vielmehr versuchen wir, falls sich eine attraktive Alternative anbietet, das Risiko aktiv zu beeinflussen, z.B. mit einer Impfung oder durch den Abschluss einer Versicherungspolice. Mit anderen Worten: Bei einer Risikoentscheidung spielt das Risikomanagement eine zentrale Rolle. Wie Massnahmen zum Risikomanagement gesucht und unter welchen Umständen sie in eine Alternative eingebaut werden, ist eine 
interessante Frage, die mit der klassischen Entscheidungstheorie nicht modelliert werden kann.

\section{Wissenschaft, Medizin, Wirtschaft}

Und wie ging es mit dem Zeichnen von Karikaturen weiter? «Dies war immer ein Teil meines Lebens. Während dem Studium zeichnete ich beispielsweise für die Studentenzeitung», sagt Oswald Huber. Und fügt an: «Dabei konnte ich gut experimentieren und lernen, welche Zeichnungstechniken sich am besten für Publikationen eignen.» Dass der Cartoonist Huber dabei Fortschritte machte, zeigt sich an der breiten Palette von Publikationen, in denen seine Zeichnungen später regelmässig zu sehen waren. Dazu gehören unter anderem die $F A Z$, die NZZ am Sonntag und das Spektrum der Wissenschaft.

Im Zuge der Neuausrichtung vieler Verlagshäuser fielen regelmässig erscheinende Karikaturen dem Spardruck zum Opfer. So publiziert der heute 76-jährige Huber «nur» noch regelmässig in der $S \ddot{A} Z$ und der Medical Tribune.

Etwas ist Huber während seiner Zeit als Cartoonist treu geblieben: In seinen Zeichnungen nimmt er Themen aus Wirtschaft und Wissenschaft aufs Korn. «Politische Karikaturen kommen für mich nicht in Frage. Bei diesen Themen ärgere ich mich viel zu oft.»

Und wo holt sich der Karikaturist seine Inspirationen? «Vor allem Schlagzeilen und Schlagworte bringen mich auf neue Ideen", so Huber. Diese lassen in seinem Kopf Bilder entstehen, die der Zeichner dann zuerst mit Bleistift und danach mit Tinte aufs Papier bringt. Dabei möchte Oswald Huber immer eines: «Die Betrachter zum Lachen und zum Nachdenken bringen.» Dafür müsse er in jeder Karikatur einen Stolperstein einbauen - dies sei denn auch die grosse Kunst und Herausforderung für jeden Cartoonisten.

\section{Kulturschock und Röstigraben}

Und wie beurteilt Oswald Huber als gebürtiger Österreicher, der schon bald vierzig Jahre in der bilinguen Stadt Fribourg arbeitet und lebt, die Schweiz? «Am Anfang erlitt ich einen Kulturschock. Vor allem weil man hierzulande viel freier ist», erinnert sich Huber. Für uns selbstverständliche Details wie, dass man den Zeitpunkt, wann man seine Steuererklärung einreichen will, selbst bestimmt oder die Krankenkasse frei wählen darf, imponierten Oswald Huber zu Beginn seiner Schweizer Zeit. Und natürlich die Sprachenvielfalt. Beeinflusst der viel beschworene Röstigraben seine Themenwahl beim Zeichnen? «Nein, im Bereich der Wissenschaft existiert der Röstigraben nicht», winkt Huber ab. Wichtig sei jedoch, dass die kurzen Dialoge in seinen Werken möglichst treffend übersetzt würden.
Wir dürfen gespannt sein, welche Themen Oswald Huber künftig in der $S \ddot{A} Z$ aufgreift. Nur so viel: Zurzeit faszinieren ihn Beiträge rund um Drohnen.

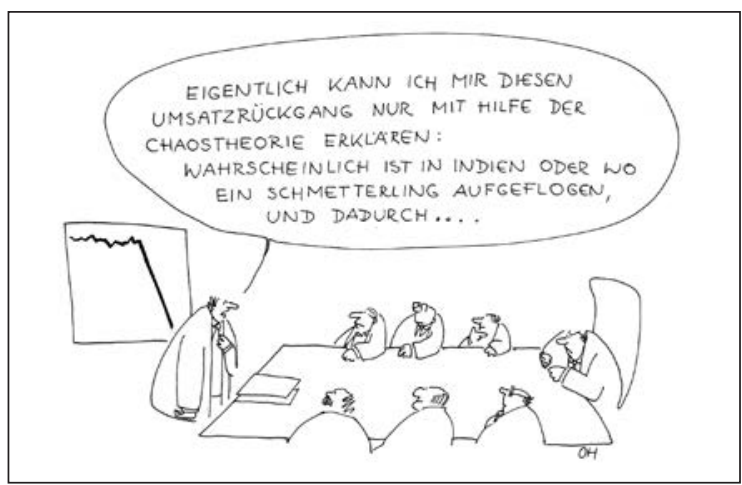

Publiziert in Blick durch die Wirtschaft (1994).

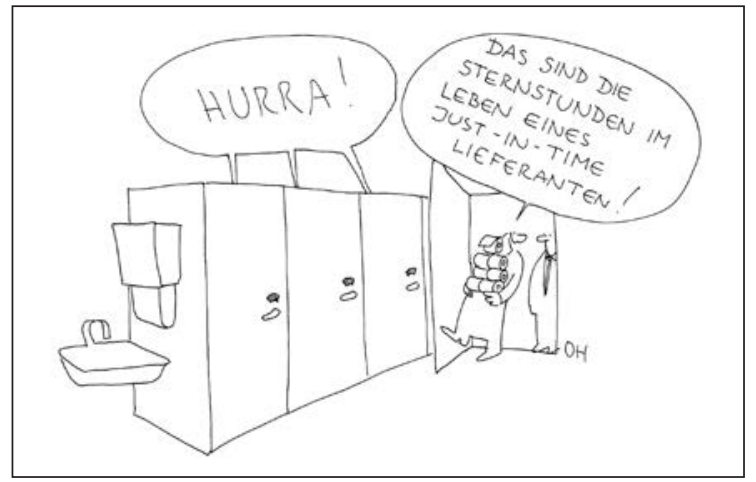

Publiziert in Blick durch die Wirtschaft (1995).

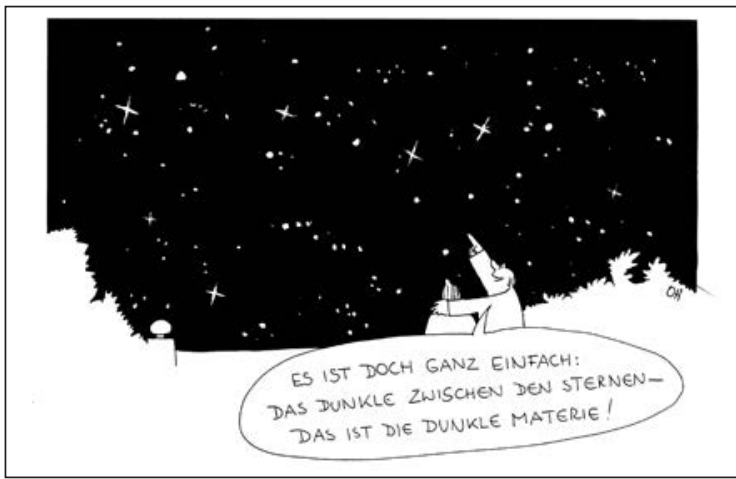

Publiziert in Spektrum der Wissenschaften (2008).

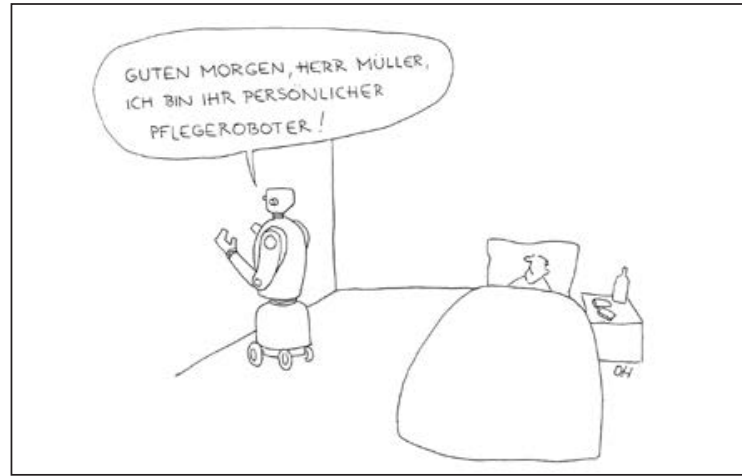

Publiziert in der SÄZ (2018).
Korrespondenz: mscholer[at]emh.ch 